\title{
The Research and Construction of Virtual PLC Laboratory based on Configuration Software
}

\author{
Zhang Yuyan* \\ Shenyang Institute of Engineering \\ Automatic Control Department \\ Shenyang, China \\ zhangyy@sie.edu.cn \\ * Corresponding Author \\ Han Xichang \\ Shenyang Institute of Engineering \\ Automatic Control Department \\ Shenyang, China \\ Hanxc@sie.edu.cn
}

\author{
Zhang $\mathrm{Na}$ \\ Shenyang Institute of Engineering \\ Automatic Control Department \\ Shenyang, China \\ zhangna@sie.edu.cn
}

\begin{abstract}
The controlling objects of PLC were simulated by configuration software, and the virtual PLC Laboratory based web environment was built using the web publishing capabilities of configuration software. In this article, Taking Zijin Bridge configuration software as a platform, a Siemens s7-200 PLC as a control device, verifies the feasibility of virtual PLC laboratory construction based on Web access. The designed thoughts, network, function construction and designed procession were described in detail. This system provides a virtual environment similar to the actual experiment for students. In this experiment, students can operate remotely the designed experiment, and the experimental data and results can be observed at the same time. Students can learn experimental project purpose, principles and related information by system help. Teachers can monitor and evaluate experiment results. This scheme solves the difficulties of the PLC traditional experimental methods. The system is low cost, maintenance-free, flexible, strong commonality and high security etc.
\end{abstract}

Keywords-PLC; Configuration Software; Virtual Experiment; Design Thoughts; Realization

\section{INTRODUCTION}

Traditional PLC (Programmable Logic Controller, PLC) experiments adopt PLC equipment and related operation panels, whose biggest flaw is that we can only observe the current state of the output points instead of the concrete operation process. Therefore it lacks visualization and humanization. And for the fact that PLC experiment mostly uses the hardware circuit structures of the object, so there are several shortcomings [1-3]: hardware devices occupy a large amount of laboratory space, hardware is expensive and difficult to modify and upgrade, hardware maintenance difficulties, etc. So, how to carry out the new automation transformation has become an urgent problem of the experiment, and the use of modern control technology and industry for state control technology can better solve the problem [4-6].

Configuration software is specialized software with functions of data acquisition and process control [7-8].

\author{
Jiang Zefan \\ Shenyang Institute of Engineering \\ Automatic Control Department ZB121 \\ Shenyang, China \\ 2357515745@qq.com
}

They are software tool, which are in the level of automatic controlling system monitoring layer's software platform and development environment, using flexible configuration mode to provide users with quick industrial automatic control system building for monitoring function and the general levels. As well, they can support all kinds of industrial equipment and common communication protocol, at the same time, configuration software itself also provides the function of Web based remote access [9$11]$.

Therefore, combining the advantages of PLC and configuration software, establishing PLC configuration of the virtual laboratory not only solves the problems in experiment teaching, course design, practice and graduation design in Automation Major, but also saves a lot of money for the teaching and the scientific research, shortening the development cycle, creating a good economic benefit. It's a revolution in practical teaching mode.

\section{VIRTUAL PLC LAB PLATFORM DESIGN THOUGHTS}

The current development of experimental platform for virtual PLC mainly aims at specialties and technicalities of the platform. Experimental platform focusing on inspiring from basic training, fully and systematically considers the influence of design on the user, as shown in Fig. 1.

Competition consciousness can motivate people's curiosity. User rating system refers to the system, in which user can do continuous learning for higher level, in order to arouse people's competitive consciousness. Most beginners will lose interests because of the onerous and boring study. But various operating interface (game interface, animation interface, and virtual classroom mode) and BBS interaction will be a good help for the solution of the above problems. As a professional experiment platform, the authenticity and how close to the real life is a very important factor. In the process of the design of the experiment platform, the above two factors have always been the subject of the design. Parent's recognition, as a 
key factor to personal growth, also reflects in this platform design, by transforming complex special knowledge into a graphic display, crossing professional communication barriers, improving users parent recognition. This experiment platform will improve user study comparing thoughts, making learning PLC a trend, which in turn promotes parents recognition, so as to achieve purpose of motivating the user interest in PLC and raising enthusiasm of PLC study.

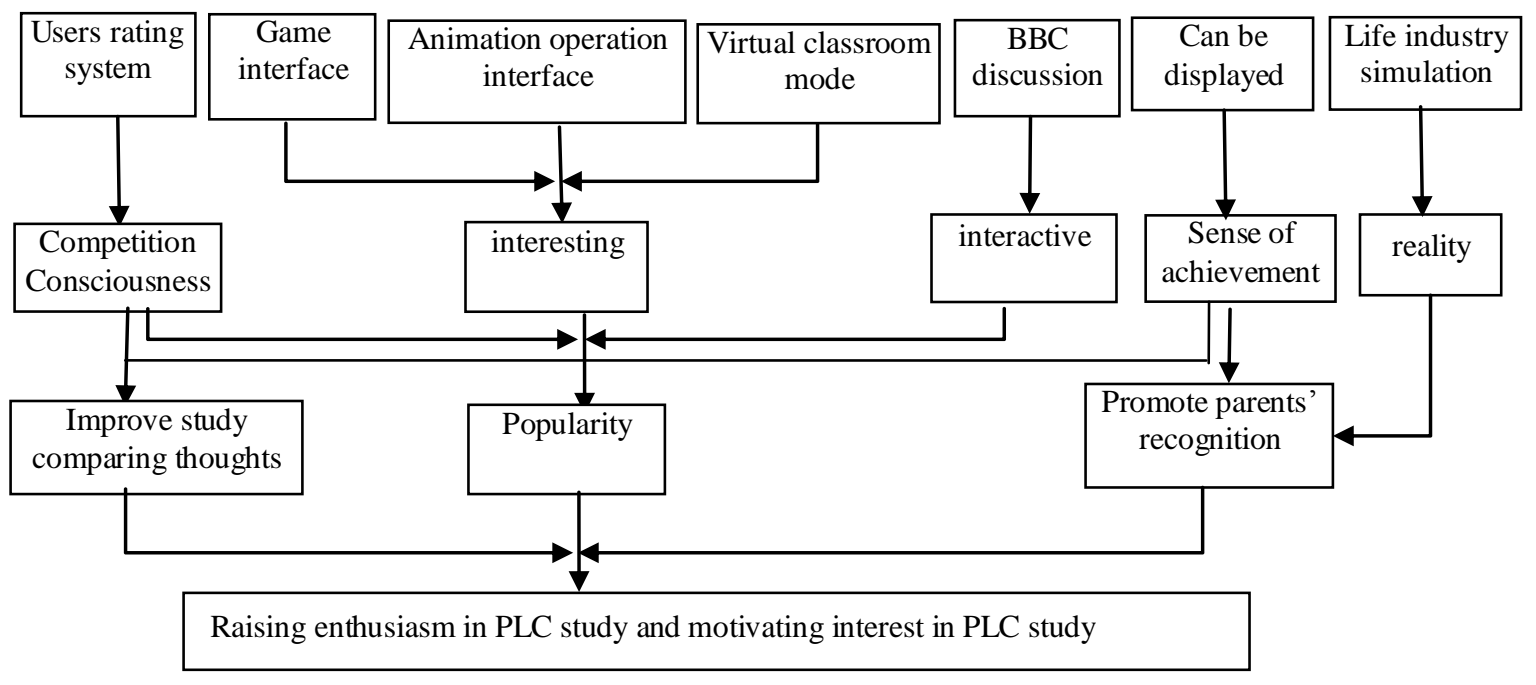

Figure 1. Virtual PLC Lab Platform Design Thoughts

\section{VIRTUAL PLC LAB DESIGN BASED ON CONFIGURATION}

\section{A. Network Design}

The system is based on Zijin Bridge configuration software as a platform to develop simulation object to control experiments with Step7-MicroWin 4.0 programming software of S7-200 PLC embedded in, to realize the aim of the control program writing. With Siemens s7-200 PLC as a control device, using the Zijin Bridge configuration software's own Web Server to realize the remote login function. Zijin Bridge communicate with PLC through the Ethernet, and it monitors all of the mesmerizers, controllers and PLC I/O interface states, transmitting to the computer in the form of a variable value, for upper machine to use and process. The network structure as shown in Fig. 2.

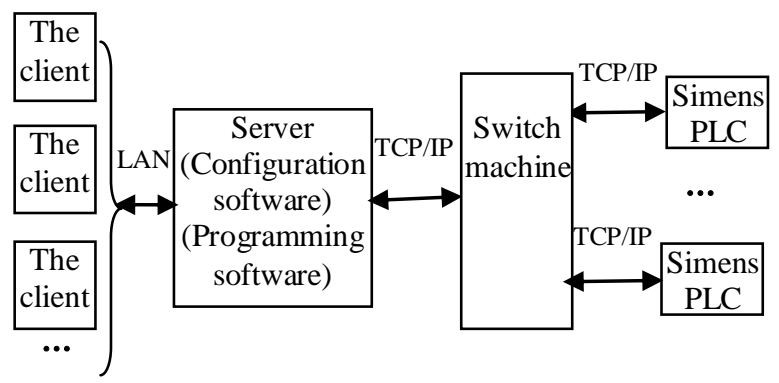

Figure 2. The Network Structure of System

\section{B. The Design of the Function Construction}

The function structure of virtual PLC experiment platform in this paper is shown in Fig. 3. The structure divides the virtual experiment into three levels, the basic training, expand training and engineering training. This kind of structure distribution can better help users learn, not only helping them learn PLC programming method from a basic level to higher, but also doing authority classifying by controlling the level of the user. The higher level users can operate more virtual experiment so that it can stimulate the user more competitive consciousness, allowing users to study harder in order to gain a higher authority. This virtuous cycle promote the user's progress in PLC study. Virtual experiment in basic training includes two plates, which are video learning and text information learning, allowing users to get rid of the bondage of original books to consult the materials, in a way that is more easily through the bidirectional query by video and text to obtain what they require. In conclusion, it can minimize the beginners' study monotony of PLC. Because it can show the diversity of configuration programming, and this platform has an on-display plate and a BBS plate for users.

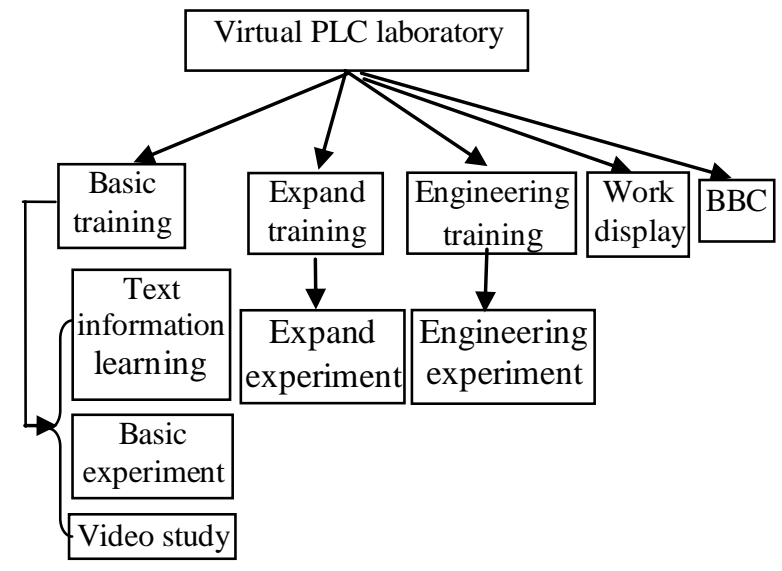

Figure 3. Function Structure of Experiment Platform 


\section{Realization of Virtual PLC Experiment System}

The basic component of virtual laboratory architecture is shown in Fig.4, mainly including four parts: interface configuration, database configuration, communication settings and remote login.

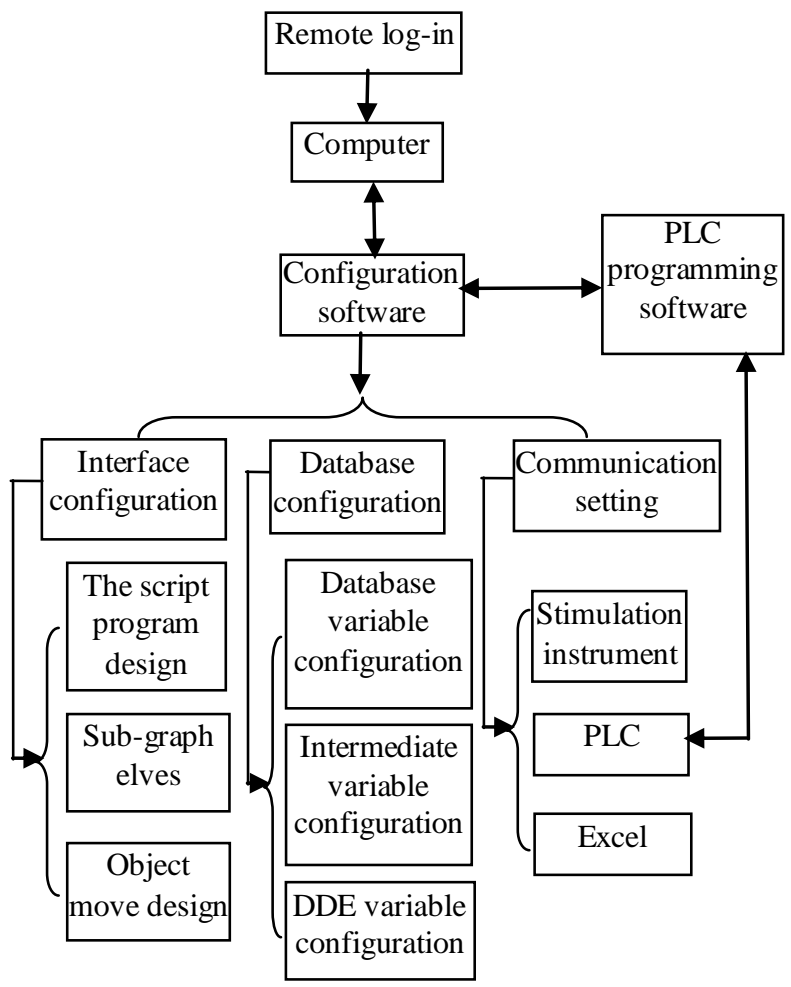

Figure 4. The Basic Component of the Virtual Laboratory

\section{1) The Development of the Graphical Interface}

The graphical interface is the embodiment of virtual experiment. Configuration software, as a software platform tool for user to customize functions, can virtualize a variety of control object. Virtual controlled object graphics can be completed by adopting drawing tools, provided by configuration software or by calling gallery. They can also be finished by the special image processing software to make virtual controlled objects have professional colors. When doing virtual objects overall planning, we should reflect the characteristics of the actual industrial control equipment and its working scene vividly, in the meantime, the input control panel (control buttons, etc.) should be reflected in order to achieve control of the virtual object. Graphical interface development mainly includes the determination of graphics, data variables, animation, connection, debugging command language and virtual object.

Making the login and registration interface as the first picture in a virtual laboratory, the user need to $\log$ in to enter the system. However, a new user must register first. Registered users' authority is divided into three levels: administrators, teachers and students. The administrator is responsible for user management, secure strategy management as well as system management and configuration; Teacher is mainly responsible for production and management of experimental teaching resources, the generation and management of the project, experimental apparatus, experimental equipment and experimental object database management and maintenance, experimental process and experimental results of monitoring and evaluation; Students log into the virtual experiment teaching system mainly to choose experiment project and therefore do the operations relating to experiment.

Logged in, click to enter into the experiment contents selection interface, in which users choose the experiment. And at every experiment interface, there are related subsidiary interface to choose.

\section{2) Establishment of the Database}

Database configuration is the core part of the virtual laboratory [9]. Signals from the communication interface and signals outputted to a configuration object are based on database configuration as the center to complete full subjects and experiment system in order to implement the open experimental effect. When doing the database configuration, a configuration point should have participatory, avoiding relations between the subject and the external control device, therefore when doing the database configuration; we only need to do a table image input and output ports, trying to minimize the impact of virtual experiment object and external devices [6].

There are three main kinds of data in the database source. One is from an external device (PLC), namely the database variables. It relates the actual PLC I/O points and PLC internal registers' change to lay the foundation for animation of the virtual devices, such as equipment startstop control and display of running status of equipment; One is a memory variable, from the simulation instrument, it is mainly used for the realization of the management functions, such as performance evaluation, error, etc.; Another is a DDE variables, from EXCEL documents, mainly used in the control system of the analog simulation, such as the simulations of water level, pipes, pressure, flow, motor current, temperature, etc.

\section{3) Communication Setting}

Configuration software in this system communicates with Siemens PLC by the TCP/IP protocol, and using DDE communication between configuration software and Excel.

\section{4)Remote Login Setting}

There are two ways of Zijin Bridge software Web publishing: using the Web Server at Zijin Bridge to publish web; And Using Microsoft IIS to do Web publishing. This system uses the Web Server at Zijin Bridge to do Web publishing. Pay attention to that in Win2000 / NT environment, due to the default boot, the system Web Server and Web Server Zijin Bridge come into conflicts. So in order to normally use Web publishing of Zijin Bridge system, Web Server functions of the system must be forbidden. In addition, to ensure the client correct access to Web Server of Zijin Bridge, IE browser security should be changed. The ActiveX and all five attributes of the plug-in should be set. 


\section{CONCLUSIONS}

Virtual laboratory is a kind of innovation laboratory itself. In the point of view of the experimental results and the experimental cost saving, it has obvious advantages. This new experimental model is bound to promote the development of college teaching. Taking Zijin Bridge configuration software as a platform, a Siemens s7-200 PLC as a control device, verifies the feasibility of virtual PLC laboratory construction based on Web access. The laboratory specific advantages as follows:

- Using configuration software to develop virtual laboratory has the characteristics of low cost, no maintenance, small experimental consumption, no afraid of damage, high security, etc. For staff training and experimental teaching, good effect can be achieved.

- Using configuration software development of the virtual laboratory has the characteristic of strong commonality. Because there is a variety of equipment to be connected to the configuration, in practice, as long as the $\mathrm{I} / \mathrm{O}$ point attribution in the virtual device configuration, selection of memory or communication mode make a little change, the development of virtual laboratory can be used to other PLCs or smart card project development or practice teaching therefore manpower, financial resources can be saved so as to greatly improve the work efficiency.

- Using configuration software to develop a virtual laboratory can directly observe the results correct or not from the interface, which brings much convenience to students' programming and debugging; Combined with advanced technology, navigation intuitive interface and good manmachine interface, students' autonomous learning interest can be improved; Virtual laboratory can provide experimenters with experiment sites at any time and at any place. As long as the experimenter can connect to the Internet, he can use a virtual laboratory experiment.

\section{ACKNOWLEDGMENT}

This work was supported in part by Liaoning Province Education Science "Twelfth Five Year Plan" project under Grant No.JG14DB292, JG15DB289 and JG15DB301, Course construction project of quality resources sharing in Liaoning Province.

\section{REFERENCES}

[1] Yong He,Guang-Lin Tian,Yi • Dan Bao,Application of networkbased Virtual Instrument Technology On Remote Vehicle Inspection,IEEE,2005: 1428-1431.

[2] W.J. Shyr, Enhancement of PLC programming learning based on a virtual laboratory $[\mathrm{J}]$.World Transactions on Engineering and Technology Education, 2010, 2(8): 196-202.

[3] Sílvia D, Olabarriaga, A virtual laboratory for medical image analysis [J].IEEE transactions on information technology in biomedicine, 2010, 4(14): 979-985.

[4] Arief Hidayat and Victor Gayuh Utomo, Virtual Laboratory Implementation to Support High School Learning [J]. International Journal of Computer Applications, 2015, 16(120):14-18

[5] Wang Xiaodong, Research and Construction of a PLC Virtual Laboratory [J]. Research \& Exploration in Laboratory, 2012, 9(31):210-213

[6] Yuan Jumei. Reform and Practice of Electrical and PLC Control Technology Teaching[J].China Educational Technology \& Equipment, 2015, 6:95-97.

[7] Liang Tingkui. Application of iFix Configuration Software in Virtual Controlled Object $[\mathrm{J}]$. Mechanical Engineering \& Automation, 2011, 03: 197-199.

[8] Wang Xiaodong, Research and Construction of a PLC Virtual Laboratory $[\mathrm{J}]$. Research \& Exploration in Laboratory, 2012, 9(31):210-213

[9] Zhang Jie, Liu Yishun and Li Jingpeng. Control System for Virtual Elevator Based on Configuration Software [J]. Machinery \& Electronics. 2010, 01:72-74.

[10] Yuan Yunfeng. On PLC Virtual Object Designed Based on iFIX Configuration Software[J], Journal of Yueyang Vocational and Technical College, 2012,01: 90-92.

[11] Zhang Guanghong,Shi Suwen. Development of Virtual Device based on PLC and Configuration Software [J]. Modular Machine Tool \& Automatic Manufacturing Technique, 2009, 09: 29-32. 